F López Cantos, J Millán Yeste (2018): “La difusión de discursos pseudocientíficos en la radio pública española. El programa Complementarios de RNE-Radio 5”. Revista Latina de Comunicación Social, 73, pp. 317 a 330. http://www.revistalatinacs.org/073paper/1257/16es.html

DOI: $\underline{10.4185 / R L C S-2018-1257}$

\title{
La difusión de discursos pseudocientíficos en la radio pública española. El programa Complementarios de RNE-Radio 5
}

\author{
Diffusion of pseudoscientific discourses in Spanish public radio. \\ The program Complementarios by RNE-Radio 5
}

\begin{abstract}
Francisco López Cantos [CV] [ ORCID] [ G GS]. Professor in Audiovisual Communication and Advertisement. Universidad Jaume I de Castellón - flopez@uji.es
\end{abstract}

J. Millán Yeste $[\underline{\mathrm{CV}}]$ Collaborator of the research group on Communication and Scientific Culture. Universitat Jaume I de Castellón - $\underline{\text { al341301@uji.es }}$

\begin{abstract}
s
[ES] Introducción. En esta investigación vamos a analizar los contenidos del programa radiofónico Complementarios de la cadena pública Radio 5 como muestra de la preocupante pandemia de discursos pseudocientíficos que se distribuyen desde los medios de comunicación. Metodología. Utilizamos el análisis de contenido desde la perspectiva de la Frame Theory con el fin de determinar el dispositivo discursivo con que promueven los contenidos pseudocientíficos en este programa radiofónico. Resultados. La cadena pública de radio pretende promover un espacio divulgativo y de utilidad social, pero contribuye en la difusión de contenidos sin ninguna validez científica y se pone al servicio del discurso pseudocientífico. Discusión. Las denominadas terapias complementarias y los discursos pseudocientíficos ocupan el espacio público aparentando ser útiles y científicamente válidos. Conclusiones. Estos contenidos mediáticos pueden resultar muy nocivos y derivar en un serio problema de salud pública y habría que tratarlos desde una perspectiva mucho más crítica y científicamente responsable.

EN] Introduction. In this research we analyze contents of the radio program Complementarios of the public chain Radio 5 as a sample of the concerning pandemic of pseudoscientific discourses broadcast by media. Methodology. We used content analysis from the Frame Theory perspective to determine the discourse device with which pseudoscientific contents are promoted in this radio program. Results. The public radio chain aims to promote an informational space and of social utility but contributes instead to the diffusion of contents without any scientific validity and puts itself at the disposal of the pseudoscientific discourse. Discussion. The so called complementary therapies and pseudoscientific discourses occupy the public space pretending to be useful and scientifically valid. Conclusions. These mediatic contents can be quite harmful and derive into serious public health problems, and should be treated from a much more critical and scientifically responsible perspective.
\end{abstract}




\section{Keywords}

[ES] Terapias complementarias; terapias alternativas; pseudociencias; tratamiento informativo; radio. [EN] Complementary therapies; alternative therapies; pseudosciences; informative treatment; radio.

\section{Contents}

[ES] 1. Introducción. 2. Metodología 3. Resultados 3.1. Estrategia de impostación y participación de expertos 3.2. Marcos interpretativos y de razonamiento promovidos. 3.3. Dispositivo comunicativo y retórica discursiva. 4. Discusión y conclusiones 5. Notas 6. Referencias bibliográficas

[EN] 1. Introduction. 2. Methodology 3. Results 3.1. Setting strategy and participation of experts 3.2. Promoted interpretative and reasoning frames. 3.3. Communicative device and discursive rhetoric. 4. Discussion and conclusion 5. Notes 6. List of references

Translation by Yuhanny Henares

(Academic translator, Universitat de Barcelona)

\section{Introducción.}

Hoy día se sigue debatiendo qué es la ciencia desde versiones más o menos evolucionadas de la escuela popperiana que buscan un concepto de verdad a partir del falsacionismo lógico (Popper, 1973); o desde aproximaciones, en el otro extremo, herederas del anarquismo epistemológico de Feyerabend (1978) que niegan en mayor o menor medida tal posibilidad de verdad. Otras concepciones contemporáneas de la ciencia como el modelo de paradigmas científicos (Kuhn, 1962) o las propuestas de revisión del positivismo (Lakatos,1983) están bien aceptadas por la comunidad científica y, de un modo u otro, establecen líneas de demarcación entre lo que se considera ciencia y lo que no lo es. En cualquier caso, identificar y desenmascarar las denominadas falsas ciencias o pseudociencias resulta en muchas ocasiones una tarea compleja de la que se han venido ocupando a lo largo de las últimas décadas desde filósofos como Mario Bunge hasta el conocido mago azote de charlatanes y estafadores Jame Randi y divulgadores científicos de la talla de Martin Gardner, Michael Shermer o el popularmente conocido Carl Sagan, quienes han ido caracterizando aquellas prácticas que se considera que tienen poco o nada de científicas o que son, simplemente, alucinaciones más o menos ingeniosas o claras estafas (vid. López-Cantos, 2017).

Lo cierto es que la preocupante pandemia contemporánea de pseudociencias que invade las redes y los medios de comunicación desvirtúa la práctica científica de manera muy significativa al usurpar su estatus, manipulando las emociones de las audiencias y causando un creciente embrutecimiento y retroceso cultural (Alonso-Marcos \& Cortiñas-Rovira, 2014), y encuentra su caldo de cultivo en un entorno comunicativo en el que, como denuncia Elías (2013), se permite que cualquiera pueda editar y reelaborar la información de un experto y manipularla para hacerla creíble. Así, las pseudociencias hacen un uso eficiente de las estrategias retóricas y las tecnologías comunicativas contemporáneas, en combinación con el frecuente recurso a apoyos y credenciales aparentemente científicas en busca de legitimidad y superioridad moral y epistemológica frente a la que denominan ciencia oficial, y como resultado de ello hoy día la utilización de mecanismos de contención destinados a limitar la expansión de esta pandemia de creencias y estafas, y la propia determinación de la autoridad científica, resulta una tarea de gran complejidad por su propia heterogeneidad (Fasce, 2017).

Uno de los ámbitos en los que las pseudociencias resultan más nocivas y tiene más prevalencia es el de las áreas de salud en las denominadas terapias complementarias y alternativas que se presentan 
como "propuesta de cura de enfermedades, alivio de síntomas o mejora de salud, basada en criterios sin el respaldo de la evidencia disponible” (OMC, 2016).

La denominada medicina complementaria o alternativa, integra un conjunto de disciplinas terapéuticas que se presentan a sí mismas como respuesta a las carencias de las instituciones y el sistema de salud convencional sin que exista garantía epistémica alguna acerca de su eficacia. A pesar de ello, no solo continúan siendo introducidas bajo distintas denominaciones en algunos centros médicos, sino que, además, y a pesar de su probada ineficacia terapéutica, se siguen promoviendo y apoyando de manera incondicional por parte de algunos medios de comunicación, incluso aquellos que son financiados con fondos públicos, como ocurre en el programa Complementarios de Radio 5. Y cuando las pseudociencias se difunden con total normalidad y sin crítica alguna en los medios de comunicación se institucionalizan y, por extensión, adquieren legitimidad a partir de la credibilidad supuesta a los medios de comunicación (Armentia, 2002) y, en consecuencia, un poder discursivo notable para estructurar la realidad haciendo uso de los poderosos efectos que los medios de comunicación tienen sobre los individuos y la sociedad a largo plazo (McQuail, 1985).

Estos discursos terapéuticos que promueve la pujante industria de la felicidad y están cada vez más presentes en todos los medios de comunicación impulsan una nueva ideología cultural que impregna todos los ámbitos públicos y privados y que profundiza aún más en el actual modelo de sociedad postcapitalista contemporánea en red y hedonista y de alto riesgo sistémico que bien han caracterizado Baunman (2013), Illouz (2008), Beck (2002), Castells (1997), Giddens (1994) o Lipovetsky (1986, 2007). Y así, estas nuevas corrientes discursivas en torno a promesas de mejora y felicidad están conformando lo que se viene denominando cultura psicoterapéutica (Rieff, 1966; Furedi, 2004; Illouz, 2007), cuyos orígenes se encuentran en los movimientos NewAge de los años 60 y 70 . Las contemporáneas pseudoterapias de la felicidad están fundamentadas en gran medida en discutibles propuestas de la nueva corriente de la psicología positiva que promueven Seligman $(1991,2002)$ y Csikszentmihalyi (1990) y tiene entre sus más reputadas gurús a Fredrickson (2009). Y, aunque el debate acerca de la definición de felicidad y lo que se puede considerar una buena vida hunde sus orígenes en la tradición del pensamiento griego, estos nuevos discursos psicoterapéuticos no solo obvian la profunda reflexión filosófica que se ha hecho al respecto si no que, además tienen nula evidencia científica.

En cuanto a la difusión que se hace de este tipo de discursos pseudocientíficos en los medios de comunicación, que es lo que nos concierne en este artículo, cabe tener en cuenta el diferente matiz con que se define la objetividad en la práctica periodística y científica, en tanto que "journalists think objectivity demands 'trying to let the facts speak for themselves', and academics think it requires systematic methods and transparent accounts” (Post, 2015: 731). Pero que la práctica periodística esté sujeta a particulares reglas temporales y ritmos de producción de contenidos (Resnik, 1998) no exime a los profesionales de los medios de comunicación de su responsabilidad ética.

En este trabajo de investigación nos vamos a ocupar de determinar las características y estrategia discursiva del programa Complementarios partiendo de la hipótesis principal (H1) de que en el programa de la radio pública se hace un tratamiento poco profesional e irresponsable de los contenidos que se difunden. Y, para ello, vamos a analizar la procedencia de sus fuentes y la estrategia discursiva seguida en la elaboración del programa para la construcción de veracidad y credibilidad desde el punto de vista comunicativo y periodístico.

\section{Metodología}

En primer lugar, analizamos con detalle la trayectoria académica y profesional de los colaboradores que participan en el programa Complementarios, para contextualizar la validez científica de sus 
declaraciones y aportaciones. A partir de ello, analizaremos el contenido discursivo de una muestra de tres programas que se han seleccionado de manera aleatoria.

Los programas emitidos hasta el momento de redacción de este artículo muestran en sus títulos, ya por sí solos, el tipo de contenidos que difunden en línea con el discurso pseudoterapéutico que hemos venido comentando y promueven en la radio pública española, y con total impunidad, prácticas que han sido catalogadas como pseudoterapias por la Organización Médica Colegial de España (OMC, 2016), tales como la acupuntura o la $P N L$, y algunas otras tan disparatadas como la descodificación dental o los cantos védicos, entre otras técnicas que giran en torno a diversas formas de coaching y de promesas de felicidad tan propias de la cultura psicoterapéutica que venimos denunciando y con nula validez científica y terapéutica:

- Canto védico y reuniones familiares navideñas

- Educar para amar la vida

- La acupuntura y las relaciones familiares

- Ser feliz en el trabajo

- Buscamos la felicidad con Elsa Punset

- Joe Dispenza

- "Más allá de la materia", de Félix Torán

- Gimnasia consciente y cómo gestionar los miedos

- Inteligencia financiera y terapia corporal

- Eneagrama y técnicas para hablar y escuchar mejor

- Coaching nutricional y comunicación

- $\quad$ El método Ikigai y Pallapupas

- Christian Beyer y la descodificación dental

- Sonidos terapéuticos y coaching familiar

- El cerebro emocional y el arte de educar con amor

- Hospital de Tarrasa y Demián Bucay

Los contenidos seleccionados para análisis corresponden a los siguientes programas emitidos en Radio5:

- $\quad$ Hospital de Tarrasa y Demián Bucay.10 de septiembre de 2017.

- $\quad$ El método Ikigai y Pallapupas. 8 de octubre de 2017.

- $\quad$ Eneagrama y técnicas para hablar y escuchar mejor. 22 de octubre de 2017.

El análisis se realiza desde la perspectiva de la pragmática lingüística y la teoría de los actos de habla propuesta por Austin (1962) y completada por Searle (1969) que distingue entre enunciados: representativos (comprometen al hablante con la veracidad de la proposición expresada); directivos (intentan intervenir en la conducta del oyente); compromisorios (comprometen al hablante en la realización de un acto futuro); expresivos (expresan un estado psicológico que se especifica en la condición de sinceridad); y declarativos (provocan cambios inmediatos en la situación institucional). En este sentido, los enunciados presentes en los eventos que se analizan están enmarcados en lo que podríamos denominar actos de habla representativos y compromisorios, en tanto que enuncian y dan por válida una verdad preexistente e inciden en las consecuencias futuras que tienen los actos enunciados.

Desde esta perspectiva teórica, y utilizando la metodología del Frame Analysis (Goffman, 1974) y entendiendo que el proceso de framing "essentially involves selection and salience" (Entman, 1993: 
52), elaboramos un mapa de palabras clave, frases hechas y símbolos dentro de los marcos interpretativo, o frame devices, y de razonamiento, o reasoning devices (Gamson \& Modigliani, 1989), para así determinar el tipo de interpretación que se promueve entre los oyentes en el programa Complementarios y constatar el grado de cumplimiento de nuestra hipótesis de partida (H1), es decir, el tratamiento poco profesional e irresponsable de los contenidos por su nula validez científica.

\section{Resultados}

El programa radiofónico Complementarios lleva en antena en Radio 5 desde el 10 de septiembre de 2017 y se emite cada domingo de 10:35 a 11 de la mañana en la cadena pública española. Según se afirma en la presentación del propio programa: “Complementarios es una nueva propuesta de Radio 5 con la que pretendemos daros ideas para vivir mejor. Con menos estrés. Con más serenidad. Con más sentido. Para ello os presentamos a profesionales de técnicas como la meditación, el mindfulness, el yoga, la acupuntura, el coaching o la PNL, entre muchas otras."[1]

Y semana tras semana las conductoras y a la vez directoras del programa, Luisa Segura Albert, Soraya Rodríguez Contreras y Cristina Serrat, exponen una terapia de carácter pseudiocientífico, remarcando desde el inicio del primer episodio que "se trata de herramientas que en ningún caso sustituyen ni contradicen a la medicina ni a la ciencia, sino que, en su caso, las complementan”. Todas ellas son licenciadas en Periodismo pero, además, tanto Soraya Rodríguez como Cristina Serrat se han formado en el Instituto Gestalt de Barcelona en especialidades pseudocientíficas tales como Programación Neurolingüística (PNL), Coaching Wingwawe e Hipnosis Eriksoniana, entre otras titulaciones académicas. [2]

\subsection{Estrategia de impostación y participación de expertos}

Los colaboradores habituales y los expertos invitados al programa muestran un posicionamiento discursivo fundamentado con claridad en el intento sistemático de legitimar este tipo de tratamientos que denominan alternativos o, como reza el título del programa, Complementarios. El modelo argumentativo está fundamentado en las aportaciones científicas de profesionales y expertos con cierto prestigio por su cargo o su actividad que, además, se dedican a promover las más variopintas pseudoterapias que, a veces, ilustran con testimonios, como en el caso de la doctora del Hospital de Tarrasa y su paciente tratado con coaching nutricional y medicina energética: [3]

\begin{tabular}{|c|c|c|}
\hline $\begin{array}{c}\text { CRISTINA } \\
\text { ABADÍA }\end{array}$ & $\begin{array}{l}\text { Doctora de la } \\
\text { unidad médica } \\
\text { integrativa del } \\
\text { hospital de } \\
\text { Tarrasa. }\end{array}$ & $\begin{array}{l}\text { Las terapias complementarias y alternativas } \\
\text { no curan, simplemente mejoran la calidad de } \\
\text { vida del paciente. A pesar de admitir que NO } \\
\text { son ciencias, quiere demostrar el } \\
\text { funcionamiento de estas terapias con } \\
\text { procedimientos científicos. }\end{array}$ \\
\hline $\begin{array}{l}\text { MARIONA } \\
\text { TAPIONA }\end{array}$ & $\begin{array}{l}\text { Paciente de la } \\
\text { unidad de } \\
\text { medicina } \\
\text { integrativa el } \\
\text { hospital de } \\
\text { Tarrasa. }\end{array}$ & $\begin{array}{l}\text { Las terapias complementarias y alternativas a } \\
\text { las que se ha sometido (medicina energética y } \\
\text { coaching nutricional) le han ayudado a } \\
\text { eliminar las dolencias de la quimioterapia. } \\
\text { Recomienda estas terapias a otras personas } \\
\text { con su situación. }\end{array}$ \\
\hline
\end{tabular}

Fuente: elaboración propia

En el mismo programa interviene el director y responsable de prensa de las charlas TEDx Barcelona que no hace otra cosa que promocionar su evento con el argumento del piscolabis posterior (en relación 
al dispositivo discursivo y las estrategias de marketing de las TEDTalks así como su validez científico/educativa se puede consultar López-Cantos, 2018). Finalmente, en el programa se incluye una entrevista a Demián Bucay, psiquiatra argentino, hijo del conocido Jorge que, como no puede ser de otra manera, es continuador de su simplista discurso de carácter motivacional con nula validez pedagógica:

\begin{tabular}{|c|c|l|}
\hline Demián Bucay & $\begin{array}{c}\text { Psiquiatra } \\
\text { (argentino) }\end{array}$ & $\begin{array}{l}\text { Educar supone generar autonomía, no hacer } \\
\text { cumplir unas normas. Para ello es necesaria la } \\
\text { motivación como elemento clave de la } \\
\text { educación. }\end{array}$ \\
\hline
\end{tabular}

Fuente: elaboración propia

En otro de los programas analizados se nos brinda información sobre el método de moda, el Ikigai,[4] que, ¡cómo no!, procede de Oriente, y así se nos presenta un autor con su libro que gira en torno a este pastiche filosófico que a los occidentales nos aportará una sabiduría ancestral que nos hará más felices y longevos.

\begin{tabular}{|c|c|c|}
\hline \multirow{7}{*}{ Francesc Miralles } & $\begin{array}{c}\text { Periodista y } \\
\text { especialista en } \\
\text { el ámbito de la } \\
\text { psicología. } \\
\text { Coautor del } \\
\text { libro El } \\
\text { método Ikigai }\end{array}$ & $\begin{array}{l}\text { Los habitantes de la región japonesa de la } \\
\text { cual procede el método Ikigai, tienen una } \\
\text { vida más longeva por sus hábitos de vida } \\
\text { basados en la felicidad, la amistad y la } \\
\text { motivación por vivir. }\end{array}$ \\
\hline
\end{tabular}

Fuente: elaboración propia

Este programa continúa con otras de las pseudoterapias que tienen más predicamento, la risoterapia, candidata perfecta a TEDx [5] y en línea con esta pandemia de psicología positiva que parte de los trabajos ya comentados de Seligman y está acabando por consolidar su institucionalización académica en forma de cátedras de dudosa calidad científica en algunas de nuestras universidades y colonizando los discursos mediáticos:

\begin{tabular}{|c|c|l|}
\hline & $\begin{array}{c}\text { Fundadora de } \\
\text { Pallapupas y } \\
\text { participante de } \\
\text { las charlas } \\
\text { AEDie Rosales }\end{array}$ & $\begin{array}{l}\text { La risa aporta ventajas al cerebro, siendo } \\
\text { incluso posible reír en situaciones } \\
\text { complicadas. }\end{array}$ \\
& La muerte como hecho humano y natural. & \\
\hline \multirow{2}{*}{ Fuente: elaboración propia }
\end{tabular}

El tercer programa analizado se dedica casi por completo a una de las más disparatadas pseudoterapias de moda, el eneagrama, [7] con la aportación experta de quien a sí mismo se autodefine como terapeuta, para continuar con otra aportación de una de las técnicas alternativas de moda que sus promotores pretenden implementar, y lo están haciendo con éxito para sus bolsillos, en distintas áreas que van desde el ámbito empresarial al educativo o el familiar. 


\begin{tabular}{|c|c|l|}
\hline Javier Muro & $\begin{array}{c}\text { Terapeuta } \\
\text { especialista en } \\
\text { el Eneagrama }\end{array}$ & $\begin{array}{l}\text { El eneagrama ayuda a conocernos mejor y } \\
\text { mejorar conociendo aquellos caracteres que } \\
\text { nos faltan. }\end{array}$ \\
\hline Sonia Ferrer & $\begin{array}{c}\text { Experta en } \\
\text { Coaching } \\
\text { familiar }\end{array}$ & $\begin{array}{l}\text { Saber comunicarnos amablemente con } \\
\text { nuestros seres cercanos y escucharlos nos } \\
\text { hará más felices. }\end{array}$ \\
\hline
\end{tabular}

Fuente: elaboración propia

\subsection{Marcos interpretativos y de razonamiento promovidos.}

Para determinar los marcos interpretativos que se ofrecen a los oyentes del programa Complementarios hemos examinado e identificado, siguiendo el método de análisis deductivo propuesto por Entman, "the presence or absence of certain keywords, stock phrases, stereotyped images, sources of information and sentences that provide thematically reinforcing clusters of facts or judgments" (Entman, 1993: 52). Y, con posterioridad, hemos agrupado los términos en torno a categorías interpretativas, o frame devices en la terminología utilizada por Gamson y Modigliani "that condense information and offer a 'media package' of an issue" (Gamson \& Modigliani, 1989), y que denominamos en lo sucesivo (Fn).

En el programa Complementarios, el discurso promueve con claridad un marco interpretativo, o framesetting en palabras de De Vreese (2005:52), de las terapias complementarias y alternativas como ciencias curativas (F1):

- Mariona Tapiona: “Tras dos sesiones de medicina energética me desaparecieron las dolencias”

- Doctora Abadía: "Queremos demostrar su funcionamiento con procedimientos científicos, generar evidencia científica.”

- Angie Rosales: "Reír tiene ventajas para el cerebro. Dicen que disminuye muchísimo el estrés, libera endorfinas, te predispone a una actitud más positiva y activa la sexualidad. [...] Hay mucha bibliografía que habla del tema”.

En casos aislados se limitan los efectos curativos de estas pseudoterapias, pero recurriendo a las mejoras en el bienestar y la promoción de estados de mayor felicidad (F2).

- Doctora Abadía: “Las terapias complementarias y alternativas no curan, simplemente mejoran la calidad de vida del paciente."

- Conductoras del programa: "Las TCA son herramientas que en ningún caso sustituyen ni contradicen a la medicina ni a la ciencia, solamente ayudan a vivir mejor."

- Francesc Miralles: "Unos hábitos de vida basados en la felicidad, la amistad y la motivación por vivir alargan la esperanza de vida."

Es habitual el recurso retórico a la parábola, es decir, a narraciones con objetivos moralistas, con reminiscencias claras de carácter pseudorreligioso y asociadas a las propuestas NewAge en torno al descubrimiento y la reflexión interior como solución a los estados de salud o anímicos:

- Un santuario muy especial (cuento): las personas construimos grandes mentiras de la nada por el simple hecho de imitar los actos del vecino y no indagar en los orígenes del asunto.

- Los eruditos (cuento): uno puede decir muchas cosas de palabra, pero después no ser lo que está presumiendo ser. 
- Doctora Abadía: “Acompañamos en el sufrimiento”, “Intentamos que tengan una buena enfermedad”, "La enfermedad es una invitación a reflexionar sobre nuestro modelo de vida”.

- Mariona Tapiona: "Me sentí muy bien acompañada y mimada”.

- Francesc Miralles: “El Ikigai es aquello que te motiva a levantarte por la mañana.”

En algunos casos se utiliza el recurso a metáforas que son bien conocidas en el ámbito de las pseudoterapias, y las pseudociencias en general, y que proporcionan un particular marco interpretativo estructurante, tal como es definido por Lakoff y Johnson (1980), como en este ejemplo en que se asocia la luz con la inteligencia y con las mejoras en el estado general del ser:

- Javier Muro: “El eneagrama es un método para personas que quieran iluminarse”, "Nos ayuda a desarrollar estados superiores del ser, la esencia y la iluminación”

Es también recurrente que los invitados ejemplifiquen con sus propias vivencias los beneficios de las terapias que van a presentar, lo cual no indica validez alguna más allá del testimonio personal de supuesta autoridad que se atribuye a cualquiera, sea experto o no, por el mero hecho de ser protagonista del programa en un medio de comunicación (Armentia, 2002):

- Mariona Tapiona, por ejemplo, explica cómo tras dos sesiones de medicina energética le desaparecieron las dolencias de la quimioterapia. Relata su experiencia positiva afirmando que se sintió muy bien acompañada, mimada y mejoró en calidad de vida.

El análisis pormenorizado de los términos significativos muestra que los marcos interpretativos están condicionados por la subjetivación del ser (F3) tanto de los síntomas de su mal estado como de sus formas de mejora, siguiendo una estrategia argumentativa que, como decíamos, se implementa en actos de habla de carácter representativo y compromisorio utilizando términos tales como "náuseas", “pérdida de apetito” “dolencias”, “ansiedad”, “depresión”, “fatiga”, "muerte", "enfermedad", etc. y dando como solución y resultado a tal diagnóstico "efectividad", "esencia", "terapia", "felicidad", "amistad" y "motivación".

A veces los beneficios obtenidos pretenden fundamentarse sobre una base científica, aunque esta sea desconocida y no reconocida por investigación alguna por parte de la comunidad científica, o simplemente se indican sin más como mejoras para todos los males basadas en la mera creencia de que funcionan o en esa falsa autoridad comentada de quienes dicen que funcionan.

- Doctora Cristina Abadía: “El 100\% de los pacientes han reducido la ansiedad, depresión, náuseas, fatiga y han aumentado su apetito".

- Angie Rosales: "reír tiene ventajas para el cerebro. Dicen que disminuye muchísimo el estrés, libera endorfinas, te predispone a una actitud más positiva y activa la sexualidad... Hay mucha bibliografía que habla del tema”.

- Demián Bucay: (y después de dudar y tartamudear a la hora de defender con pruebas su teoría de la educación) incluye en su discurso frases como "yo creo que funciona".

- Francesc Miralles: al tiempo que promueve la sabiduría del Ikigai por su procedencia ancestral hace afirmaciones como que "El horóscopo puede estar bien si se hace con profundidad, como el eneagrama”.

\subsection{Dispositivo comunicativo y retórica discursiva}

En cuanto al dispositivo comunicativo sonoro, el programa Complementarios recurre habitualmente, como decíamos, a la parábola y siempre acaba con la lectura de un cuento de corte orientalista por parte de Cristina Serrat. Estas narraciones siempre están acompañadas con efectos de sonido de fondo 
(agua corriendo y gongs), con el evidente objetivo de que esos recursos comunicativos inviten a sumergirse en esa atmosfera orientalista que supuestamente promueve la meditación y la reflexión y que, con ese fin, aún se alargan más de un minuto y medio tras acabar la narración. En similar sentido, la voz de Cristina Serrat, con un tono suave y pausado, intenta cautivar al oyente y sumergirlo en la narración.

Uno de los cuentos narrados, sirva como ejemplo, es Un santuario muy especial perteneciente a las historias de Nasreddin, un personaje de la tradición sufí cuyas narraciones sirven para ilustrar las enseñanzas de esta doctrina religiosa, [8] y esta estrategia retórica basada en la parábola se repite igualmente también, por ejemplo, en el relato de Los eruditos. Paradójicamente, las enseñanzas de estos cuentos podrían resultar en una dura crítica a los propios contenidos del programa pues invitan al radioyente a investigar por su propia iniciativa sobre el tema y desconfiar de todo aquello que no conozcamos a la perfección. Sin embargo, el dispositivo retórico funciona, más que destinado al fomento de una actitud escéptica y crítica hacia el conocimiento en general propia de la tradición filosófica, como la simple invitación a abrazar las nuevas propuestas alternativas y complementarias que promueve el programa. Con este fin, se utiliza una pirueta retórica, y que podemos considerar cercana a las propuestas filosóficas de Feyeraben, destinada a poner en cuestión todo discurso en sí mismo y promover una crítica individual y personalista, es decir, una opinión individual fruto de los propios descubrimientos. Y con esta operación retórica se promueve conculcar por completo los mecanismos de validación científica del conocimiento tales como el contraste de datos, la reproductibilidad y el consenso de resultados y conclusiones equiparando, así, la validez de los discursos pseudocientíficos con la de los científicos.

\section{Discusión y conclusiones}

En definitiva, y en síntesis, los resultados de nuestra investigación nos permiten concluir que el programa Complementarios de Radio 5 pretende crear un espacio divulgativo y de utilidad social pero, sin embargo, lo que hace es promover pseudociencias, algunas de ellas consideradas muy peligrosas y utilizadas "con objetivos de manipulación del pensamiento en movimientos de carácter sectario" como la terapia de la Gestalt (OMC, 2016), o sin ningún valor terapéutico.[9]

Con la pretensión de dar "ideas para vivir mejor. Con menos estrés. Con más serenidad. Con más sentido" se difunden por las ondas de Radio Nacional de España - Radio 5 pseudoterapias como la meditación, el mindfulness, el yoga, la acupuntura, el coaching o la PNL, todas ellas con nulo rigor científico. Los contenidos del programa no se analizan ni contrastan críticamente en ningún caso y, bien al contrario, se intenta justificar la validez de las terapias presentadas con el mero recurso a las vivencias y aseveraciones sin ningún fundamento científico de los propios colaboradores e invitados que van pasando por este inefable programa en las sucesivas entregas.

La formación académica de las propias conductoras del programa y su contrastada y excelente trayectoria profesional, así como los duros años de formación en medicina de la Doctora Abadía, parece no eximir, ni siquiera atenuar, el mágico atractivo que tienen las terapias complementarias y alternativas en todo tipo de personas, incluso en aquellas a quienes se les supone una formación científica más que suficiente para abordar con propiedad y críticamente este tipo de pseudoconocimiento, tal como ha puesto de manifiesto Michael Shermer (1997) en su famoso libro We people belive weid things. [10] Uno de los últimos programas difundidos por Complementarios está dedicado nada menos que a las Flores de Bach, [11] una pseudoterapia fundamentada en unos principios químicos que no pueden resultar más que risibles para cualquier estudiante de bachillerato.

El resto de quienes intervienen en los programas analizados son pacientes satisfechos o tienen también formación específica como expertos de diversas técnicas y corrientes pseudocientíficas. Es muy 
preocupante que en un programa de la radio pública las presentadoras promuevan un discurso pretendidamente periodístico que, conculcando las buenas prácticas profesionales, se elabora desde la única perspectiva de los estudios que han cursado en el Instituto Gestalt de Barcelona, y cuyos miembros son invitados habituales al programa, [12] a pesar de que la terapia de la Gestalt está considerada una psicoterapia "ampliamente utilizada con objetivos de manipulación del pensamiento en movimientos de riesgo sectario" (OMC, 2016), y el resto de programas formativos en pseudoterapias que se imparten en dicho centro y similares repartidos por toda la geografía nacional no tienen ninguna validez científica. [13]

En cuanto al marco interpretativo sobre el que se despliega el dispositivo discursivo se han identificado tres frames fundamentales que presentan las pseudoterapias como ciencias curativas (F1), que sirven para las mejoras del bienestar y felicidad (F2) y son aplicables por medio de la subjetivación del ser (F3) en tanto necesitado de tratamiento y a partir de la identificación de síntomas para, de este modo, promocionar los beneficios terapéuticos de las pseudociencias presentadas.

Desde el punto de vista comunicativo, el discurso retórico que presenta el programa Complementarios hace uso a recursos sonoros que colaboran a elaborar una narración sonora de corte orientalista que culmina con parábolas cuya moraleja pretende enseñar a las audiencias que lo adecuado es determinar la validez del discurso, sea del tipo que sea pero especialmente el que se pretende parecer al científico y que denominamos pseudocientífico, a partir de la experiencia personal y con la mera investigación individual, obviando así las más básicas premisas aceptadas por la comunidad científica para la validación del conocimiento y, por extensión, pretendiendo que el conocimiento elaborado con rigor y laboriosamente por la comunidad de investigadores es tan válido como las ocurrencias y opiniones de cualquiera de los invitados al programa y las técnicas que presentan.

En definitiva, y tal como muestran los resultados de nuestro análisis, la hipótesis de partida (H1) con la que abordábamos esta investigación se cumple y demostramos que la cadena pública de radio pretende promover un espacio divulgativo y de utilidad social pero, en lugar de ello, el dispositivo discursivo y los recursos públicos se ponen al servicio de la pseudociencia y lo único que hace la cadena pública es contribuir, de manera totalmente irresponsable, en la difusión de contenidos pseudocientíficos, y que en algunos casos pueden resultar incluso peligrosos.

Las conclusiones de nuestro trabajo son concurrentes con las críticas al modelo de sociedad y la cultura terapéutica que se está imponiendo (Illouz, 2008) y ya se ha puesto en evidencia en otras investigaciones previas al respecto (Elías, 2013; Alonso-Marcos \& Cortiñas-Rovira, 2014; Cano-Orón, 2016; López-Cantos, 2017) que, de manera similar, muestran las estrategias comunicativas con que las denominadas terapias complementarias y los discursos pseudocientíficos ocupan el espacio público aparentando ser útiles y científicamente válidos. Sin embargo, son contenidos que pueden resultar muy tóxicos y un problema de salud pública y, en caso de que hayan de tener cabida en los medios de comunicación, especialmente los públicos, habría, cuanto menos, que tratarlos desde una perspectiva mucho más crítica y científicamente responsable.

\section{Notas}

1. http://www.rtve.es/alacarta/audios/complementarios/

2. Se puede consultar el detalle al respecto en https://www.linkedin.com/in/soraya-rodriguezcontreras-17042226/ y https://es.linkedin.com/in/cristina-serrat-400b451b 
3. La identificación de nuevas pseudoterapias resulta una ardua tarea para el investigador dada la rapidez con que se crean nuevos términos que multiplican así las diversas denominaciones con las que se promueven prácticas sin ninguna validez científica, la Asociación para Proteger al Enfermo de Terapias Pseudocientíficas-APETP recopila una lista que se va actualizando regularmente, http://www.apetp.com/index.php/lista-de-terapias-pseudocientificas/

4. El método Ikigai es la última moda proveniente de Japón en cuanto a recetas milagrosas para obtener la felicidad, para más detalles respecto a su fórmula mágica se puede consultar https://isabelsierra.es/2017/11/05/ikigai-la-filosofia-vida-los-japoneses-llegan-los-100-anos/

5. Las charlas TEDx son la versión local de las populares TEDTalks y se organizan por medio de una red de franquiciados y, aunque siguen las directrices impuestas por la organización TED, suelen a veces acoger temáticas y ponentes de dudosa credibilidad, véase por ejemplo http://www.lr21.com.uy/tecnologia/1348309-homeopatia-pseudociencia-tedx-rio-de-la-plataciencia

6. La charla TEDx de Angie Rosales se puede consultar en https://youtu.be/JOvMr_OxtjA

7. El eneagrama es un sistema de clasificación de la personalidad humana en 9 tipos diferentes o "eneatipos" que establece relaciones predefinidas de rechazo o afinidad entre ellos, algo que ya conocemos de la astrología, y que desde luego no tiene ninguna validez científica tal como se desprende de los análisis llevados a cabo en el seno del GRECC-Grup de Recerca en Comunicació Científica de la Universitat Pompeu Fabra, http://infopseudociencia.es/eneagramade-la-personalidad/

8. El sufismo es una doctrina religiosa ascética y mística propia del islamismo que se define como heterodoxa y panteísta e intenta conectar con Alá mediante los actos interiores y no mediante ritos y tradiciones.

9. Entre otras pseudoterapias consideradas peligrosas se encuentra la biodescodificación o la medicina germánica. Sus características se pueden consultar en el catálogo de terapias de la mente y el cuerpo elaborado por la OMC y disponible en http://www.cgcom.es/técnicas-de-la$\underline{\text { mente-y-el-cuerpo }}$

10. Se puede consultar al respecto su charla TED https://www.youtube.com/watch?v=44aK0n4MiF8, y también esta breve entrada del blog Naukas, desde el que se realiza una encomiable labor de divulgación científica de calidad y muy recomendable en todo caso, http://naukas.com/2011/01/26/1996/

11. Flores de Bach es, según se recoge en el catálogo de pseudociencias elaborado por el GRECC, el "nombre con el que se denomina un conjunto de 38 preparados artesanales no farmacológicos, elaborados a partir de una maceración en agua de flores maduras de diversas especies vegetales silvestres o naturalizadas de la región de Gales y la Inglaterra contigua, diluida en brandy (destilado del vino, usado como medio conservante) destinado a aliviar desequilibrios psicoemocionales y de carácter (tales como miedo, impaciencia, angustia, incertidumbre, ira, confusión, intolerancia, timidez, entre otros). Los principios de preparación de estos remedios son similares a los de la homeopatía, aunque su preparación se basa en diluciones más bajas. Los estudios han demostrado la ausencia de principios activos en las plantas usadas y, por tanto, su capacidad de producir efectos resulta nula. Por otro lado, estos preparados contienen una 
proporción importante de alcohol, que se usa como conservante y que puede contribuir a cierto efecto placebo.", http://infopseudociencia.es/flores-de-bach/

12. Por ejemplo en el programa del día 22 de Octubre interviene Javier Muro o en el del día 26 de Noviembre Fuensanta García, ambos ahora integrantes del que han denominado Instituto Javier Muro desde el que desarrollan su actividad como trainers, coachs y terapeutas y promueven pseudoterapias como el ya mencionado eneagrama https://www.linkedin.com/in/fuensantagarcia

13. En pro del buen hacer periodístico y de la promoción del servicio público de calidad no estaría de más que en los programas se contase con la presencia de científicos que puedan aportar otras perspectivas, que hay muchísimos, por ejemplo en lo que nos ocupa se puede consultar el trabajo que viene realizando Eparquio Delgado, http://www.eparquiodelgado.com/ o José Miguel Mulet, http://jmmulet.naukas.com/

\section{Referencias bibliográficas}

F Alonso-Marcos \& S Cortiñas-Rovira, (2014): "La pseudociencia y el poder de los medios de comunicación. La problemática ausencia de bases teóricas para afrontar el fenómeno", en Historia y comunicación social, 19, pp. 93-103.

J Armentia (2002): "Ciencia vs pseudociencias", en Mediatika. Cuadernos de Medios de Comunicación, 8. Disponible en http://www.euskonews.com/0030zbk/gaia3001es.html.

J Austin, (1962): How to Do Things with Words. Cambridge (Mass.): Harvard University Press.

Z Baunman \& D Lyon (2013): Vigilancia líquida. Barcelona: Paidós.

U Beck (2002): La sociedad del riesgo. hacia una nueva modernidad. Barcelona: Paidós.

M Bunge (2010): Las pseudociencias. ¡Vaya timo! Pamplona: Laetoli.

L Cano-Orón (2016): "Correlación entre las búsquedas sobre terapias complementarias en Google y su uso por parte de la población española", en Panace@, Vol. XVII (44), pp. 124-132.

M Castells (1997): The Power of Identity. The Information Age. Economy, Society, and Culture, Volume II. Oxford: Blackwell Publishers.

M Csikszentmihalyi (1990): Flow: The Psychology of Optimal Experience. New York: Harper \& Row.

CH de Vreese (2005): "News framing: Theory and typology", en Information Design Journal + Document Design, Vol. 13(1), pp. 51-62.

C Elías (2013): "Contraconocimiento y pandemias de credulidad en la Sociedad Red: el papel del periodismo en la búsqueda de la verdad en los entornos digitales», en Estudios sobre el mensaje periodístico, Vol. 19 (2), pp. 667-681.

RM Entman (1993): "Framing: Toward Clarification of a Fractured Paradigm", en Journal of Communication, Vol. 43 (4), pp. 51-58.

A Fasce (2017): "Los parásitos de la ciencia. Una caracterización psicocognitiva del engaño pseudocientífico", en Theoria, Vol. 32 (3), pp. 347-365

P Feyerabend (1978): La Ciencia En Una Sociedad Libre. México: Siglo veintiuno editores. 
BL Fredrickson (2009): Positivity. New York: Three Rivers Press.

F Furedi (2004): Therapy Culture. Cultivating vulnerability in an uncertain age. Londres, Nueva York: Routledge.

W Gamson \& A Modigliani (1989): "Media Discourse and Public Opinion on Nuclear Power: A constructionist approach.", en American Joournal of Sociology, Vol. 95 (1), pp. 1-37.

A Giddens (1994): Consecuencias de la modernidad. Madrid: Alianza.

E Goffman (1974): Frame analysis: An essay on the organization of experience. Harvard University Press.

E Illouz (2007): Intimidades congeladas. Las emociones en el capitalismo. Buenos Aires, Madrid: Katz.

E Illouz (2008): Saving the Modern Soul: Therapy, Emotions, and the Culture of Self-Help. California University Press.

TS Khun (1962): The Structure of Scientific Revolutions. Chicago: Chicago University Press.

I Lakatos (1983): La metodología de los Programas de investigación científica. Madrid: Alianza Editorial.

G Lakoff \& M Johnson (1980): Metaphors We Live By. Chicago: University of Chicago Press.

F López-Cantos (2017):"El discurso de la felicidad de las terapias alternativas en Facebook", en Razón y Palabra, Vol. 21 (3-98), pp. 381-393.

G Lipovetsky (1986): La era del vacío. Ensayos sobre el individualismo contemporáneo. Barcelona. Anagrama.

G Lipovetsky (2007): La felicidad paradójica. Ensayo sobre la sociedad de hiperconsumo. Barcelona: Anagrama.

D McQuail (1985): Introducción a la teoría de la comunicación de masas. Barcelona: Paidós.

OMC (2016): Observatorio OMC contra las Pseudociencias, Pseudoterapias, Intrusismo y Sectas Sanitarias. Organización Médica Colegial de España. Disponible en:

http://www.cgcom.es/observatorio-omc-contra-las-pseudociencias-intrusismo-y-sectas-sanitarias

K Popper (1973): La lógica de la investigación científica. Madrid: Tecnos.

S Post (2015): "Scientific objectivity in journalism? How journalists and academics define objectivity, assess its attainability, and rate its desirability”, en Journalism, Vol. 16 (6), pp. 730-749.

D Resnik (1998): "Problemas y dilemas éticos en la interacción entre ciencia y medios de comunicación”, en Quark: Ciencia, medicina, comunicación y cultura, 13, pp. 59-77. Disponible en: http://quark.prbb.org/13/013059.htm

P Rieff (1966): The triumph of the therapeutic. Uses of faith after Freud. Nueva York: Harper \& Row.

M Shermer (1997): Why people believe weird things. Pseudo-science, superstition, and other confusions of our time. New York: Henry Holt \& Cia.

J Searle (1969): Speech Acts: An essay in the Philosophy of language. Cambridge: Cambridge University Press. 
M Seligman (1991): Learned Optimism: How to Change Your Mind and Your Life. New York: Pocket Books.

M Seligman, M. (2002). Authentic Happiness: Using the New Positive Psychology to Realize Your Potential for Lasting Fulfillment. New York: Free Press.

\section{Cómo citar este artículo / Referencia normalizada}

F López Cantos, J Millán Yeste (2018): “La difusión de discursos pseudocientíficos en la radio pública española. El programa Complementarios, de RNE-Radio 5”. Revista Latina de Comunicación Social, 73, pp. 317 a 330.

http://www.revistalatinacs.org/073paper/1257/16es.html

DOI: $\underline{10.4185 / R L C S-2018-1257}$

- En el interior de un texto:

...F López Cantos, J Millán Yeste (2018: 317 a 330) ...

o ...F López Cantos et al, 2018 (317 a 330) ...

Artículo recibido el 26 de septiembre de 2017. Aceptado el 1 de febrero. Publicado el 9 de febrero de 2018 